\title{
CATASTROPHE THEORY AND URBAN PROCESSES
}

\author{
John Casti and Harry Swain \\ Research Scholars at the \\ International Institute for \\ Applied Systems Analysis \\ Schloss Laxenburg \\ A-2361 Laxenburg, Austria
}

\begin{abstract}
Phenomena exhibiting discontinuous change, divergent processes, and hysteresis can be modelzed with catastrophe theory, a recent development in aifferential topology. Exposition of the theory is illustrated by qualitative interpretations of the appearance of functions in central prace systems, and of price cycles for urban housing.
\end{abstract}

\section{Introduction}

A mathematical theory of "catastrophes" has recently been developed by the French mathematician Rêné Thom $[6,7]$ in an attempt to rationally account for the phenomenon of discontinuous change in behaviors (outputs) resulting from continuous change in parameters (inputs) in a given system. The power and scope of Thom's ideas have been exploited by others, notably zeeman $[10,11]$, to give a mathematical account of various observed discontinuous phenomena in physics, economics, biology, [4] and psychology. We particularly note the work of Amson [1] on equilibrium models of cities, which is most closely associated with the work presented here. With the notable exception of Amson's work, little use has been made of the powerful tools of catastrophe theory in the study of urban problems. Perhaps this is not surprising since the theory is only now becoming generally known in mathematical circles. However, despite the formidable mathematical appearance of the basic theorems of the theory, the application of catastrophe theory to a given 
situation is often quite simple, requiring only a modest understanding of simple geometric notions. In this regard, catastrophe theory is much like linear programming in the sense that it is not necessary to understand the mechanism in order to make it work--a fairly typical requirement of the working scientist when faced with a new mathematical tool.

Thus, our objective in this article is twofold: first, to supply a brief introduction to the basic philosophy of catastrophe theory in a form which we hope will be congenial to workers in the urban field, and second, to illustrate the applications of the theory to some classical problems in urban economic geography. Specifically, we consider an example for central place theory in which the simplest type of nontrivial catastrophe provides a satisfactory global picture of the observed developmental patterns of functions provided to the population. A second example illustrates application of one of the more complex elementary catastrophes to the issue of equilibrium residential property prices in urban land markets. Although these examples are provided primarily as qualitative illustrations of the theory, it is hoped that they may be of interest in their own right as providing an alternative and possibly more comprehensive account of the dynamics of these problems than those obtained by other methods. 
Catastrophe Theory

In this section, we present a brief discussion of the basic assumptions and results of catastrophe theory in a form useful for applications. For details and proofs, we refer to $[8,9]$.

Let $f: R^{k} \times R^{n} \rightarrow R$ be a smooth (infinitely differentiable) function representing a dynamical system $\Sigma$ in the sense that $R^{k}$ is the space of input variables (controls, parameters) while $R^{n}$ represents the space of output variables (responses, behaviors). We assume that $k \leq 5$, while $\mathrm{n}$ is unrestricted. The fundamental assumption is that $\Sigma$ attempts to locally minimize $f$. We hasten to point out that in applications of catastrophe theory, it is not necessary to know the function $f$. In fact, in most cases $f$ will be a very complicated function whose structure could never be determined. All we assume is that there exists such a function which $\sum$ seeks to locally minimize.

Given any such function $f$, if we $f i x$ the point $c \varepsilon R^{k}$, we obtain a local potential function $f_{C}: R^{n} \rightarrow R$ and we may postulate a differential equation

$$
\dot{x}=-\operatorname{grad}_{\mathrm{x}} f \quad
$$

where $x \in R^{n}, \operatorname{grad}_{x} f=\operatorname{grad} f_{C}=\left(\frac{\partial f}{\partial x_{1}}, \ldots, \frac{\partial f}{\partial x_{n}}\right)$.

Thus, the phase trajectory of $\sum$ will flow toward a minimum of $f_{c}$; call it $x_{C}$. The stable equilibria are given by the minima of $f_{c}$, and, since there are usually several minima, $x_{C}$ will be a multivalued function of $c$; that is, $x_{C}: R^{k} \rightarrow R^{n}$ is not one-to-one. The objective of catastrophe theory is to analyze this multivaluedness by means of the theory of singularities of smooth mappings.

We first state the fundamental result of catastrophe theory in relatively precise mathematical language. We then interpret each of the conclusions of the main theorem in everyday language to show their reasonableness and applicability for real-world problems.

For completeness, and to round out the mathematical theory, we consider not only the minima but also the maxima and other stationary values of $\mathrm{f}_{\mathrm{C}}$. Define the manifold $\mathrm{M}_{\mathrm{f}} \subset \mathrm{R}^{\mathrm{k}+\mathrm{n}}$ as

$$
M_{f}=\left\{(x, c): \operatorname{grad}_{x} f=0\right\}
$$

and let $X_{f}: M_{f} \rightarrow R^{k}$ be the map induced by the projection of $R^{k+n} \rightarrow R^{k}$. $X_{f}$ is called the catastrophe map of $f$. Further, let $J$ be the space of $\mathrm{C}_{\infty}$-functions of $\mathrm{R}^{\mathrm{k}+\mathrm{n}}$ with the usual Whitney $\mathrm{C}^{\infty}$-topology. Then the basic theorem of catastrophe theory (due to Thom) is the following. 
Theorem: There exists an open dense set $J_{0} \subset J$, called generic functions, such that if $f \varepsilon J_{0}$

(i) $M_{f}$ is a k-maniford;

(ii) any singularity of $x_{f}$ is equivalent to one of a finite number of elementary catastrophes;

(iii) $x_{f}$ is stable under small perturbations of $f$.

\section{Remarks :}

1. Here equivalence is understood in the following sense: maps $X: M \rightarrow N$ and $\bar{X}: \bar{M} \rightarrow \bar{N}$ are equivalent if there exist diffeomorphisms $h, g$ such that the diagram

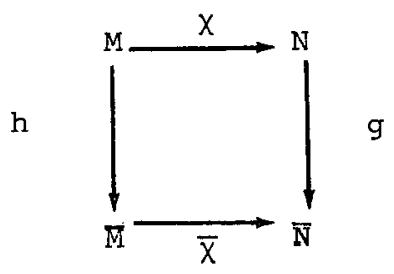

is commutative. If the maps $x, \bar{X}$ have singularities at $x \in M, \bar{x} \varepsilon \bar{M}$, respectively, then the singularities are equivalent if the above definition holds locally with $\mathrm{hx}=\overline{\mathrm{x}}$.

2. Stable means that $x_{f}$ is equivalent to $x_{g}$ for all $g$ in a neighborhood of $f$ in $J$ (in the whitney topology).

3. The number of elementary catastrophes depends only upon $\mathrm{k}$ and is given in the following table:

\begin{tabular}{ccccccc}
$\mathrm{k}$ & 1 & 2 & 3 & 4 & 5 & 6 \\
\hline $\begin{array}{c}\text { number of elementary } \\
\text { catastrophes }\end{array}$ & 1 & 2 & 5 & 7 & 11 & $\infty$
\end{tabular}

A finite classification for $k>6$ may be obtained under topological, rather than diffeomorphic, equivalence but the smooth classification is more important for applications.

4. Roughly speaking, $J_{0}$ being open and dense in $J$ simply means that if the potential function $f \in J$ were to be selected at random, then fEJ $J_{0}$ with probability one. Thus, a given system function $f$ is almost always in $J_{0}$, and furthermore, if it is not, an arbitrarily small perturbation will make it so.

5. The importance of $M_{f}$ being a $k$-manifold is that $M_{f}$ is the place where controlling influence is exerted: from the standpoint of the decision maker, $M_{f}$ is the manifold which he may manipulate. Thus, the dimension of the behavior or output space does not enter into 
the classification at all. Since $n$, the dimension of the behaviox space, may be very large, this conclusion enables us to focus attention upon a much smaller set in investigating where and when catastrophic changes in behavior will occur. To sumarize, $M_{f}$ is where the action is.

6. Conclusion ( $i$ ) shows that, mathematically speaking, only a very small number of distinctly different catastrophes can occur. Intuitively, catastrophes are equivalent if they differ only by a change of coordinate system. Since the coordinate system chosen to describe a phenomenon is not an intrinsic feature of the system, we may restrict our attention to the analysis of only a small handful of mathematical catastrophes, safe in the knowledge that more complex forms cannot possibly occur. In addition, as indicated below, the elementary catastrophes are all described by simple polynomials which make their analysis and properties particularly simple.

7. The last conclusion, stability, means that should the potential f describing $\Sigma$ be perturbed slightly, the new potential will also exhibit the same qualitative catastrophic behavior as f. Since no physical system is know precisely, this fact enables us to feel confident about various predictions based upon use of any $f \in J_{0}$. 
Discontinuity, Divergence, and the Cusp Catastrophe

Our critical assumption is that $\Sigma$, the system under study, seeks to minimize the function $\mathrm{f}$ : that is, $\Sigma$ is dissipative. Thus, the system behaves in a manner quite different from the Hamiltonian systems of classical physics. In this section we shall mention two striking features displayed by catastrophe theory which are not present in Hamiltonian systems but which are observed in many physical phenomena.

The first basic feature is discontinuity. If $\beta$ is the image in $\mathrm{R}^{\mathrm{k}}$ of the set of singularities of $\chi_{f}$, then $\beta$ is called the bifurcation set and consists of surfaces bounding regions of qualitatively different behavior similar to surfaces of phase transition. Slowly crossing such a boundary may result in a sudden change in the behavior of $\Sigma$, giving rise to the term "catastrophe". Since the dimension of the output space does not enter in the classification theorem, all information about where such catastrophic changes in output will occur is carried in the bifurcation set $\beta$ which, by a corollary of conclusion ( $i$ ) of the Theorem, is a subset of the input space $\mathrm{R}^{\mathrm{k}}$. Hence, even though $\Sigma$ may have an output space of inconceivably high dimension, the "action" is on a manifold of low dimension which may be analyzed by ordinary geometric and analytical tools.

The second basic feature exhibited by catastrophe theory is the phenomenon of divergence. In systems of classical physics a small change in the initial conditions results in only a small change in the future trajectory of the process, one of the classical concepts of stability. However, in catastrophe theory the notion of stability is relative to perturbations of the system itself (the function f), rather than just to perturbations of the initial conditions, and so the Hamiltonian result may not apply. For example, adjacent tissues in a homogeneous embryo will differentiate.

Let us now illustrate the above ideas by considering the cusp catastrophe. It will turn out that a minor modification of this catastrophe is also the appropriate catastrophe for one of the main examples of this paper, the problem of central place discontinuities.

Let $\mathrm{k}=2, \mathrm{n}=1$, and let the control and behavior space have coordinates $a, b$, and $x$, respectively.

Let $f: R^{2} \times R^{1} \rightarrow R$ be given by

$$
f(a, b, x)=\frac{x^{4}}{x}+\frac{a x^{2}}{2}+b x
$$

The manifold $M_{f}$ is given by the set of points $(a, b, x) \subset R^{3}$ where 


$$
\operatorname{grad}_{x} f(a, b, x)=0
$$

that is,

$$
\frac{\partial f}{\partial x}=x^{3}+a x+b=0
$$

The map $X_{f}: M_{f} \rightarrow R^{2}$ has singularities when two stationary values of E coalesce, that is,

$$
\frac{\partial 2_{f}}{\partial x^{2}}=3 x^{2}+a=0
$$

Thus, Equations (1) and (2) describe the singularity set $s$ of $\chi$. It is not hard to see that $s$ consists of two fold-curves given parametrically by

$$
(a, b, x)=\left(-3 \lambda^{2}, 2 \lambda^{3}, \lambda\right), \lambda \neq 0,
$$

and one cusp singularity at the origin. The bifurcation set $\beta$ is given by

$$
(a, b)=\left(-3 \lambda^{2}, 2 \lambda^{3}\right)
$$

which is the cusp $4 a^{3}+27 b^{2}=0$. Since $M_{f}$ and $S$ are smooth at the origin, the cusp occurs in $\beta$ and not in $S$. Figure 1 graphically depicts the situation.

It is clear from the figure that if the control point $(a, b)$ is fixed outside the cusp, the function $f$ has a unique minimum, while if $(a, b)$ is inside the cusp, f has two minima separated by one maximum. Thus, over the inside of the cusp, $M_{f}$ is triple-sheeted.

The phenomenon of smooth changes in $(a, b)$ resulting in discontinuous behavior in $x$ is easily seen from Figure 1 by fixing the control parameter a at some negative value, then varying $b$. On entering the inside of the cusp nothing unusual is observed in $x$; but upon further change in b, resulting in an exit from the cusp, the system will make a catastrophic jump from the lower sheet of $M_{f}$ to the upper, or vice versa, depending upon whether $b$ is increasing or decreasing. The cause of the jump is bifurcation of the differential equation $\dot{\mathrm{x}}=-\operatorname{grad}_{\mathrm{x}} f$, since the basic assumption is that $\Sigma$ always moves so as to minimize $\hat{f}$. As a result, no position on the middle sheet of maxima can be maintained and $\Sigma$ must move from one sheet of minima to the other. 


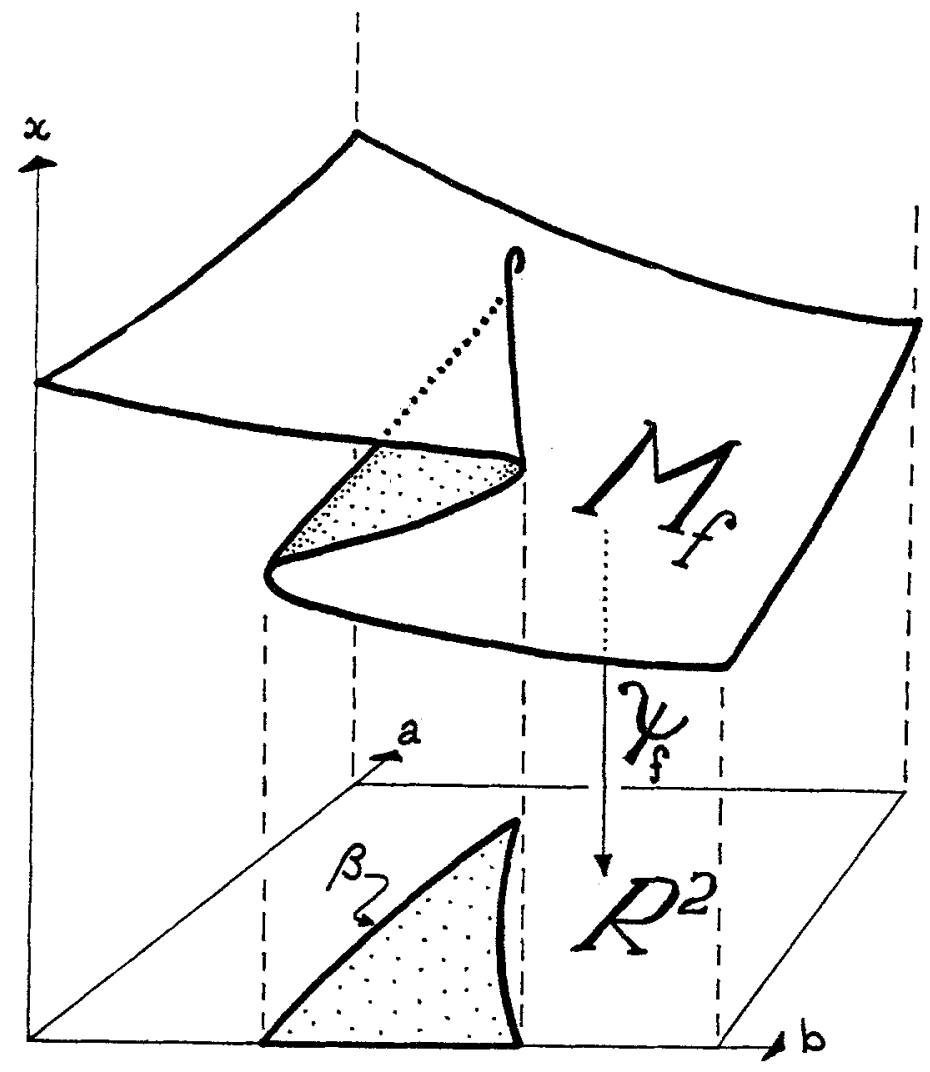

Figure 1. The Cusp Catastrophe 
A hysteresis effect is observed when moving $b$ in the opposite direction from that which caused the original jump: the jump phenomenon will occur only when leaving the interior of the cusp from the opposite side to the point of entry.

To see the previously mentioned divergence effect, consider two control points $(a, b)$ with $a>0, b \gtrless 0$. Maintaining the $b$ values fixed with decreasing $a$, the point with positive $b$ follows a trajectory on the lower sheet of $M_{f}$, while the other point moves on the upper sheet. Thus, two points which may have been arbitrarily close to begin with end up at radically different positions depending upon which side of the cusp point they pass.

While the cusp is only one of several elementary catastrophes, it is perhaps the most important for applications. In Table I, we list several other types for $k \leq 4$, but refer the reader to [6] for geometrical details and applications.

Table I. The Elementary Catastrophes for $k \leq 4$.

\begin{tabular}{|c|c|c|c|}
\hline Name & potential function $f$ & $\begin{array}{c}\text { control } \\
\text { space } \\
\text { dimension }\end{array}$ & $\begin{array}{l}\text { behavior } \\
\text { space } \\
\text { dimension }\end{array}$ \\
\hline fold & $x^{3}+u x$ & 1 & 1 \\
\hline cusp & $x^{4}+u x^{2}+v x$ & 2 & 1 \\
\hline swallowtail & $x^{5}+u x^{3}+v x^{2}+w x$ & 3 & 1 \\
\hline butterfly & $x^{6}+u x^{4}+v x^{3}+w x^{2}+t x$ & 4 & 1 \\
\hline $\begin{array}{l}\text { hyperbolic } \\
\text { umbilic }\end{array}$ & $x^{3}+y^{3}+u x y+v x+w y$ & 3 & 2 \\
\hline $\begin{array}{l}\text { elliptic } \\
\text { umbilic }\end{array}$ & $x^{3}-x y^{2}+u\left(x^{2}+y^{2}\right)+v x+w y$ & 3 & 2 \\
\hline $\begin{array}{l}\text { parabolic } \\
\text { umbilic }\end{array}$ & $x^{2} y+y^{4}+u x^{2}+v y^{2}+w x+t y$ & 4 & 2 \\
\hline
\end{tabular}


Central Place Catastrophes

To illustrate the cusp catastrophe in an urban context, consider the supply of goods and services to an urban-centered market area under all the normal postulates of classical (geometric, static, deterministic) central place theory. Then there exist spatial monopoly profits, $\pi$, in the distribution of that vast majority of goods whose threshold lies between the size of the existing market and that of the market that would be required to induce a competing supplier to locate there. The argument is similar for the number of establishments handing that good, the number of functions in a given central place, and the order of that central place (cf. Dacey [3] for definition of terms).

But not let there be emigration from that market area, or some other process producing a slow leakage of aggregate local purchasing power. Then $\pi \rightarrow 0$, the minimum threshold, at which point the good ceases to be distributed.

The threshold for (re-) appearance of the good (establishment, function) is, however, higher than $\pi=0$ since an entrepreneur would choose that combination of good and market area offering maximal spatial monopoly profits (the upper threshold). Thus we have the characteristic discontinuity and hysteresis effects of catastrophe theory.

The cusp catastrophe provides a reasonable global picture for these central place phenomena. Let the independent or control variables be $x$, the population of a market area, and $y$, the disposable income per capita. The behavior or output variable can then be interpreted as the order of the central place, or number of functions or goods provided there; all three may be generally referred to as the functional level, $\mathrm{m}$, of the central place or market area. (The implicit potential function for this system is, in contradistinction to the prior discussion, maximized by the action of the central place process. Thus we operate with $-f$ and apply the preceding theory.) The relevant picture is given in Figure 2. Each point on the manifold $M$ represents a functional level corresponding to given levels of aggregate local purchasing power. But though $\mathrm{x}$ and $\mathrm{y}$ determine the functional level, the fact that $M$ is triple-sheeted within a region near the relevant thresholds means that $m$ can take on two distinct stable equilibrium values; values, moreover, which depend on the trajectory (history or direction of change) in $x$ and $y$. Thus in Figure 2 it may be readily seen that, for a fixed level of disposable income per capita, smooth increases in population will have but small effects on the functional level of the central place until the locus of that trajectory crosses the right-hand cusp border into region II (see a). At this point the 


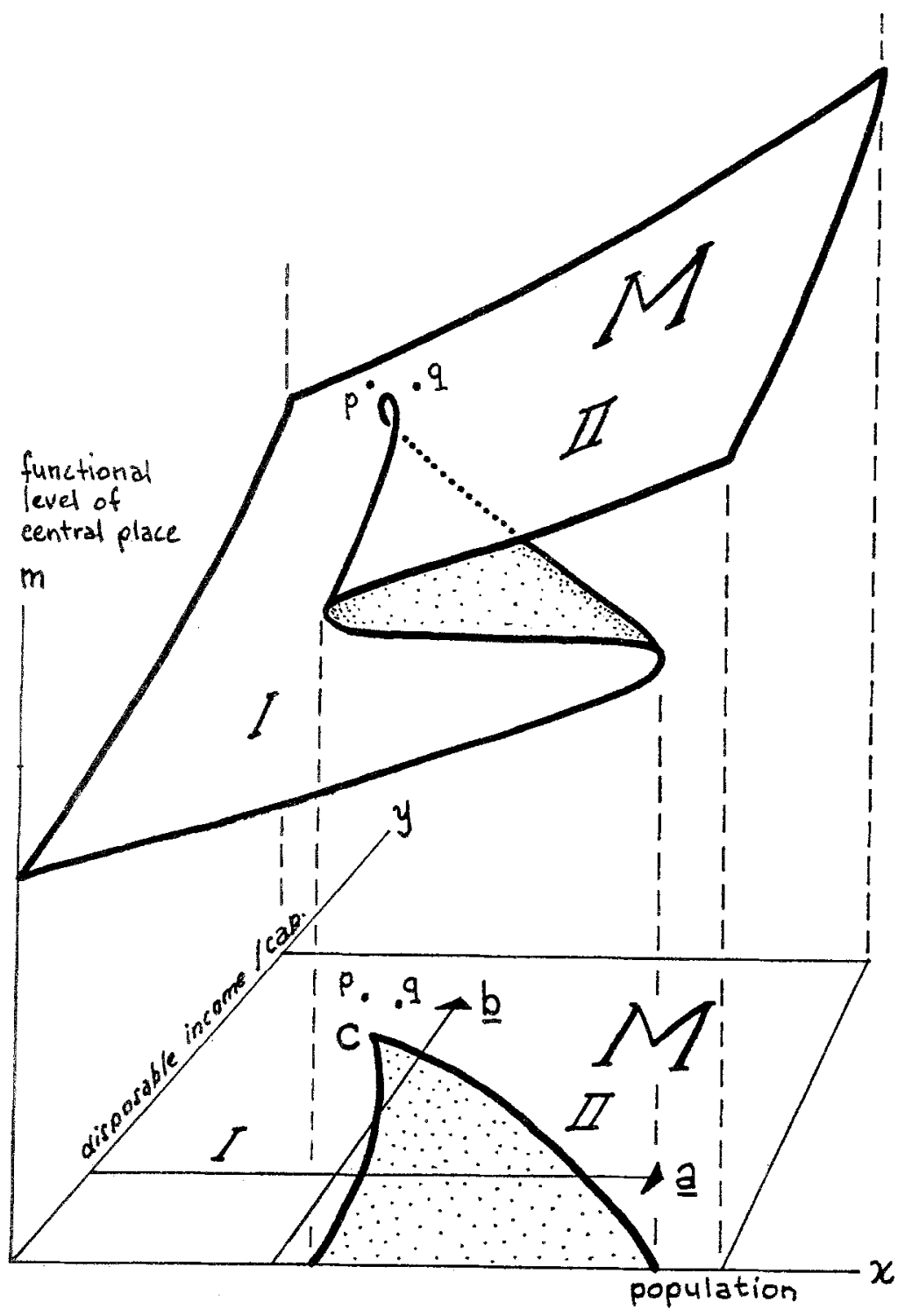

Figure 2. A Manifold for Central Place Catastrophes 
functional level jumps dramatically from the lower sheet of $M$ to the upper (the middle sheet shown in Figure 2 corresponds to relative minima and is of no interest here). The vector $\underline{b}$ shows the same qualitative result, and clearly various combinations of $\underline{a}$ and $\underline{b}$ will do the same provided such combinations pass through the $x, y$ projection of the multi-sheeted part of $M$.

The hysteresis effect can be demonstrated by examining $m$ for, say, fixed income and changing population. Let population increase along a as before; thus the cusp region is entered from $I$ and no discontinuous output; the point then leaves I and enters region II with a positive jump in functional level. But then let population smoothly decrease (-a): the cusp is entered from II at the same point as before, and the point exits into I as before. The only difference is that this time the catastrophic jump downwards in functional level takes place when entering I and not II. Only an exit from the cusp region across a different boundary than the entry branch gives rise to catastrophic change. Thus the cusp catastrophe illustrates the theoretical prediction, and observed fact, that the threshold for (re-) appearance of a function is higher than for its disappearance. Note that this qualitatively nice behavior is obtained even with the highly restrictive and unrealisitic postulates of classical central place theory. More realistic models incorporating entrepreneurial inertia (lagged feedback plus conservative behavior in the face of uncertainty), non-zero entry costs, and substantial indivisibilities would only serve to accentuate the hysteresis effect.

The third basic feature, divergence, can be appreciated by examining the change in functional level from nearby initial points $p$ and $q$ as disposable income falls for a ixed population. The trajectory in $M$ from $p$ passes to the left of the cusp point $C$, and consequently $m$ drops smoothly to levels on the lower sheet of $M$. On the other hand, the point $q$, which began with a population close to $p$, has a trajectory which takes it to the right of $\mathrm{c}$; $\mathrm{m}$ is thus maintained, for a while at least, at "artificially" or "anomalously" high levels. The critical factor is that slow change of the same sort in real regional systems with similar initial conditions may lead to fundamentally different futures, depending on the location and orientation of cusp points. Moreover, one would expect these m-anomalies to be most glaring at low levels of population and income. 


\section{Property Prices and the Butterfly Catastrophe}

The cusp catastrophe is probably useful in many other urban settings. Casual observation suggest that many of the lifestyle definition processes or our proliferating subcultures--processes noted for teenage gangs long before becoming part of the conventional wisdom about the post-industrial middle classes [2]--may exhibit the characterisitic non-Hamiltonian divergence of catastrophe theory, and may under special conditions display discontinuities and even hysteresis [5]. We discuss a more prosaic example, the purchase price of urban dwellings, not so much to exploit the cusp further but to use it as a vehicle to introduce a generalization which is perhaps the second-most-important elementary catastrophe for applied work, the so-called butterfly catastrophe.

Let $r$ represent the real rate of change of housing prices in a particular urban market. In the first approximation, we assume that there are two types of buyers who are interested in this sort of property, and that the combined level of their activities in the property market dictates $r$. Call there buyers consumers and speculators. The former are interested in a wide range of attributes of the housing bundle and their demand is strongly price-elastic, especially in volatile or cyclical markets. Speculators, on the other hand, are overwhelmingly concerned with short-term (and often highly leveraged) capital gains. Since the two groups have fundamentally different objectives, time horizons, and price elasticities, they may reasonably be thought of as disjoint sets of investors. If $D_{C}$ represents the demand for property by consumers and $D_{s}$ the demand by speculators, then the global behavior of property prices may in this simple case be as depicted in Figure 3.

Increasing either $D_{C}$ or $D_{S}$ tends to increase $r$, but the key to catastrophic rises and falls lies with the speculators; changes in $D_{C}$ for constant $D_{S}$ cause only smooth changes in $r$. All of the features observed in the previous example--divergence, discontinuity, and hysteresis--are also present here. Moreover, in empirical applications there is frequently a relation between the location of the cusp point and the time constants of the system, with loci avoiding the multisheeted parts of $M$ tending to be slower. In this example, suppose the process starts at $O^{\prime}$ in the $D_{C}-D_{S}$ space. There are then two possiblities for passage through the cusp region and back to $\mathrm{O}^{\prime}$, the paths OPQRO and OPQSO. The first corresponds to a spurt of speculative demand causing, after a short lag, a jump in prices from $P$ to $Q$, followed by a profittaking sell-off by speculators with only moderate increase in consumer demand, triggering a collapse of prices at R. This sort of process is 


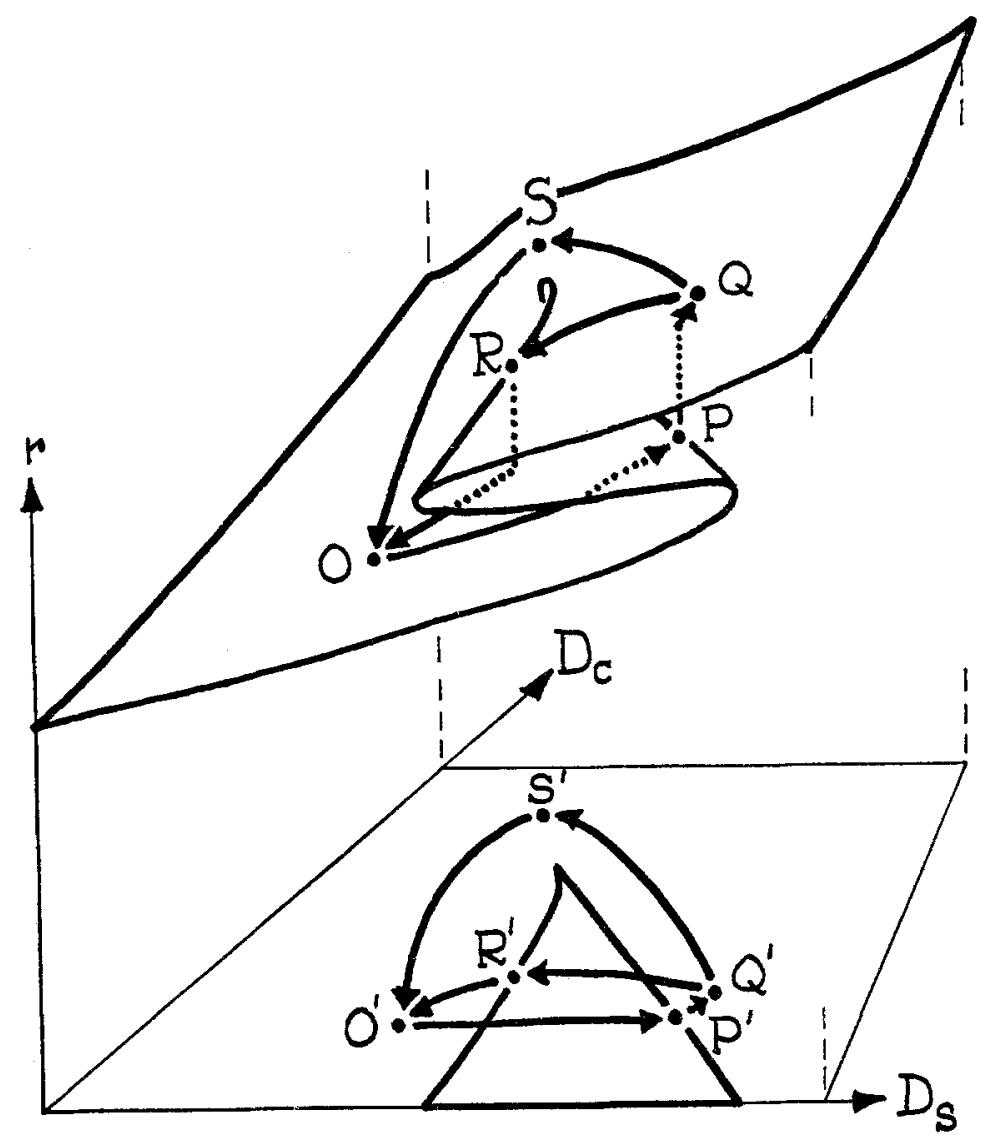

Figure 3. Catastrophe Manifold for Urban Proberty Prices 
characteristic of the high-frequency components of $r$ and is quite typical in speculative markets. The demand by consumers for market intervention is related to both the magnitude of $r$ and the amplitude of these relatively short-term "boom-and-bust" cycles. Slowing the frequency of the $O P Q R O$ cycle may be an appropriate response under such conditions, if it allows $D_{C}$ to build up sufficiently at $Q$ to drive the return path around the cusp through $s$. Rapid and distressing falls in price are thus avoided. This observation illustrates, if crudely, the fast time-slow time ("silly putty") behavior divergence which is characteristic of dynamic catastrophe models.

Governments interested in orderliness and stability in housing markets--low and viscous $r-$-usually regulate $D_{C}$ and $D_{s}$ by tightening or loosening the supply of money, that is, by raising or lowering interest rates. We now show how the butterfly catastrophe, a generalization of the cusp, enables us to upgrade the urban property price example by including time dependence as well as interest rate changes in the catastrophe manifold. It will be seen that inclusion of these important factors generates the possibility for a third mode of stable behavior of $r$, a type of "compromise" rate of change of prices.

For the butterfly $(k=4, n=1)$, the canonical form for the potential is given by

$$
f(c, x)=\frac{x^{6}}{6}+\frac{1}{4} c_{1} x^{4}+\frac{1}{3} c_{2} x^{3}+\frac{1}{2} c_{3} x^{2}+c_{4} x
$$

where $C \varepsilon R^{4}$, $x \in R$. The associated catastrophe surface $M$ is the fourdimensional surface given by

$$
\frac{\partial f}{\partial x}=x^{5}+c_{1} x^{3}+c_{2} x^{2}+c_{3} x+c_{4}=0
$$

The surface $M C R^{5}$ and the bifucation set $\beta \subset R^{4}$. We draw two-dimensional sections of $B$ to show how it generalizes the cusp. When the butterfly factor $\mathrm{c}_{1}>0$, the $\mathrm{x}^{4}$ term swamps the $\mathrm{x}^{6}$ term and we obtain the cusp. The effect of the bias factor $\mathrm{c}_{2}$ is merely to bias the position of the cusp. When the butterfly factor $c_{1}<0$, then the $x^{4}$ term conflicts with the $x^{6}$ term and causes the cusp to bifurcate into three cusps enclosing a pocket. This pocket represents the emergence of a compromise behavior midway between the two extremes represented by the upper and lower surfaces of the cusp. 


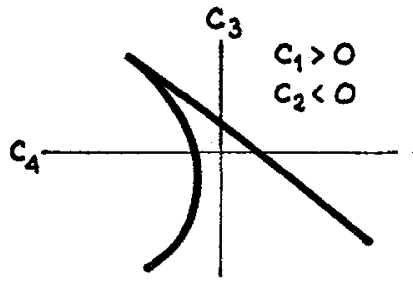

(4a)

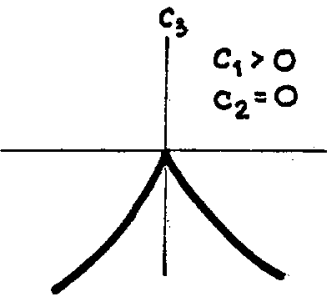

(4b)

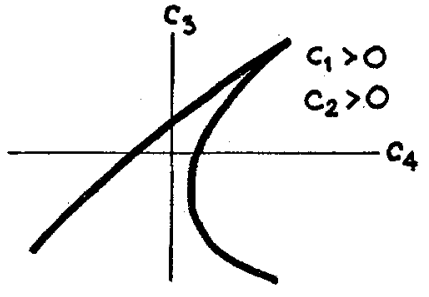

(4c)

To employ the butterfly catastrophe in the urban property price setting, we let the bias factor represent the interest rate $i$, while the butterfly factor is the negative of time, -t. Thus normalizing the nominal interest rate at $i=0$, we have the picture of Figure 5 .

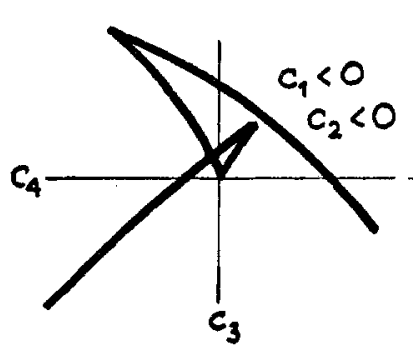

(4d)

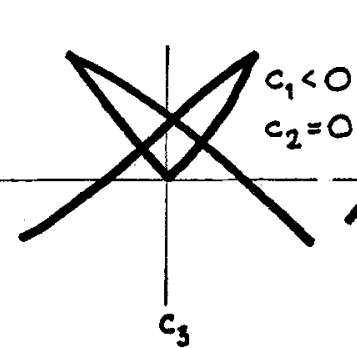

(4e)

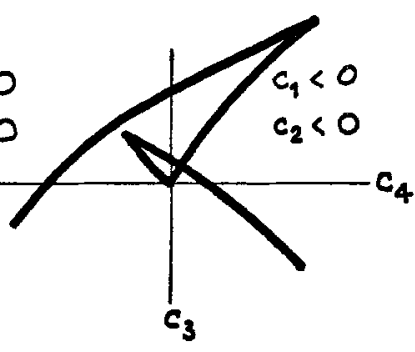

(4f)

Figure 4. Two-Dimensional sections of the Butterfly Catastrophe

Figure 5 shows that an increase of speculative demand coupled with a sufficiently high consumer demand will lead to a control space trajectory intersecting the interior pocket of intermediate $r$, rather than resulting in a dramatic jump to the upper or lower surfaces of $\mathrm{M}^{2}$. As the previous diagrams showed, manipulation of the interest rate $i$ influences both the size and position of this pocket of intermediate behavior, thereby in theory preventing catastrophic jumps or drops in property price rates--but at a price in secular inflation. 


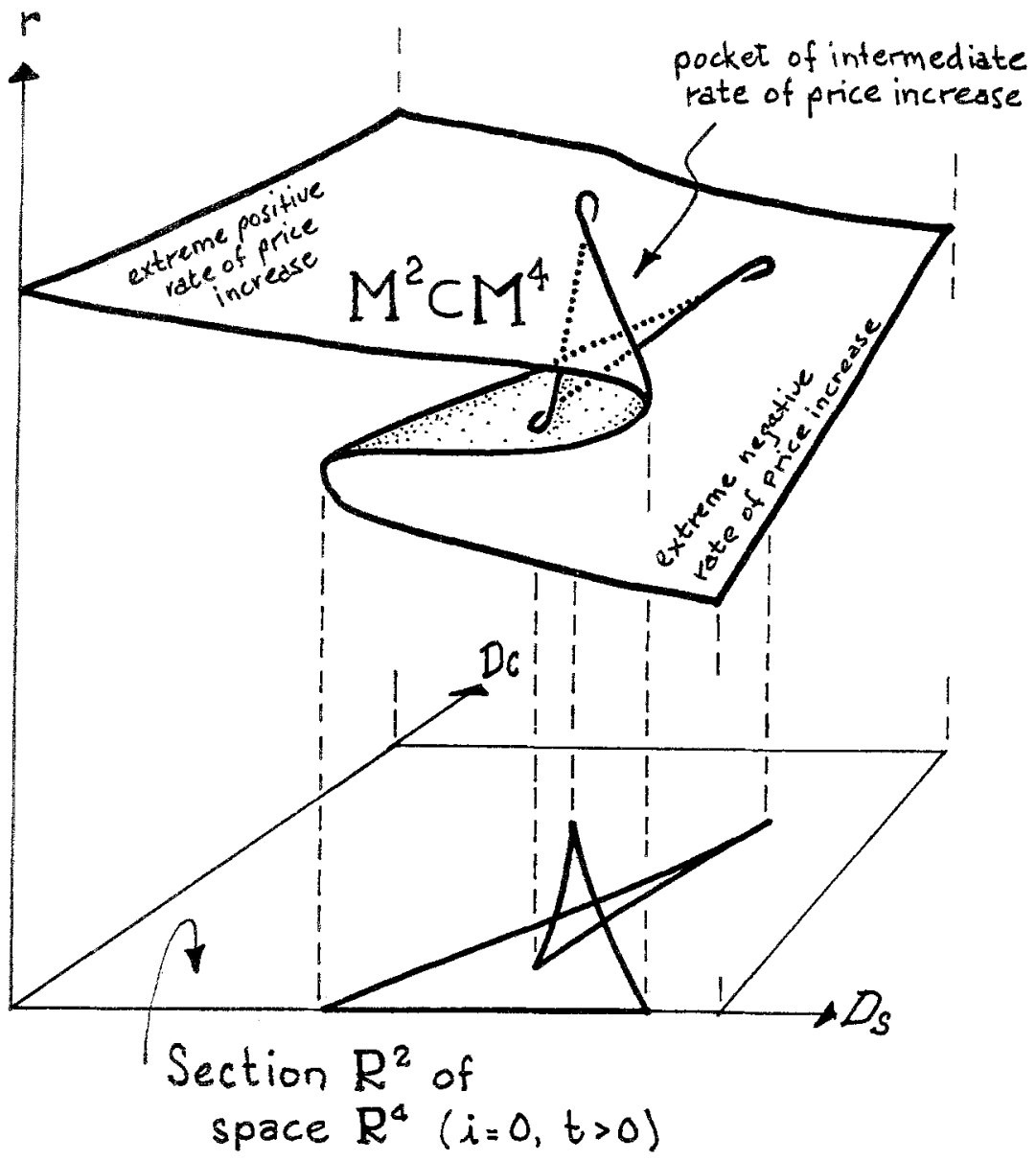

Figure 5. The Butterfly Catastrophe 


\section{Conclusions}

In this note we have presented some speculation on roles for catastrophe theory in urban studies. While the simple examples provided indicate that the mathematical theory may have something relevant to say about urban processes, it is clear that much work remains before these notions can be made into operational tools for predictive and prescriptive action. In particular, to make these ideas useful in actual decision-making contexts, the qualitative analysis given here must be made quantitative. This means the isolation cf the particular surface, or family of equivalent surfaces, pertinent to the process under study. To accomplish this task, it will be necessary to use experimental data to isolate the appropriate range of parameters which appear in the canonical potential functions. We hope to examine this circle of ideas in future work. 


\section{Iiterature Cited}

[1] AMSON, J. "Equilibrium Models of Cities: 1 - An Axiomatic Theory," Environment and Planning, 4 (1972), 429-44.

[2] BELL, D. The Coming of Post-Industrial Society: A Venture in Social Forecasting, London: Heinemann, 1974.

[3] DACEY, M.F. "The Geometry of Central Place Theory," Geografiska Annaler 47B:2 (1965), 111-24.

[4] Jones, D.D. "The Application of Catastrophe Theory to Biological Systems," paper presented at Systems Ecological Conference, Logan, Utah, 20-23 February 1975.

[5] KAHN, H. and A.J. WIENER, The Year 2000, New York: MacMillan, 1967.

[6] THOM, R. "Topological Models in Biology," Topology, 8 (1969), 313-35.

[7] THOM, R. Stabilité Structurezle et Morphogenèse, Reading, Massachusetts: Addison-Wesley, 1972.

[8] WASSERMAN, G. Stability of Unfoldings, Springer Lecture Notes in Math, vol. 393, New York: Springer-Verlag, 1974.

[9] WOODSTOCK, A.E.R. and T. POSTON, A Geometrical Study of the Elementary Catastrophes, Springer Lecture Notes in Math, vol. 373, New York: Springer-Verlag, 1974.

[10] ZEEMAN, E.C. "Differential Equations for the Heartbeat and Nerve impulse," in C. WADDINGTON, ed., Towards a Theoretical Biology, 4. Eainburgh University Press, 1972.

[11] ZEEMAN, E.C. "Applications of Catastrophe Theory," Mathematics Institute, University of Warwick, March, 1973. 